\title{
SYNTHESIS AND CHARACTERIZATION OF SILVER-
}

\section{PLATINUM NANOSTRUCTURE}

\section{MEENA DEVI $^{1} \&$ SHACHI AWASTHI ${ }^{2}$}

${ }^{1}$ Research Scholar, Department of Physics, Jayoti Vidyapeeth Women University, Jaipur, Rajasthan, India

${ }^{2}$ Faculty of Engineering and Technology, Jayoti Vidyapeeth Women University, Jaipur, Rajasthan, India

Currently, the usage of nanotechnology could be seen in the commercial market in the form of nano devices and nano materials which were playing a revolutionary part in the implications of safety, health and advancement in technology. The applications of nanotechnology could be seen in almost every field of life, resulting in making the daily life tasks so easier for the current and future generation. The nanoparticles of gold, silver and platinum could be formed with the help of the extracts of plants at the moderate temperature. However, the exploration of the fabrication of the nanostructures of Ag-Pt was not fully explored by using the tea polyphenol. Those days, the usage of nanostructures having green fabrication was noticed as it was less costly, intoxic and did not harm the atmosphere as well. Also, there were many other agents which could be used for the fabrication of nanostructures. Some of those agents were biological agents like plants, fungi and bacteria. The study was tried to explore the synthesis and characterization of silver-platinum nanostructures and its applications in the field of medicine. The purpose of this paper was to highlight the importance of teapolyphenolas a reducing agent in the synthesis of silver-platinum nanostructures. This method was quite less costly and eco-friendly.

KEYWORDS: Characterization, Nanostructures, Platinum, Silver, Synthesis \& Tea Polyphenol
\end{abstract}

Received: Feb 07, 2019; Accepted: Feb 27, 2019; Published: Mar 15, 2019; Paper Id.: IJAPBCRJUN20192

\section{INTRODUCTION}

Due to the properties like electrical, optical, magnetic and catalytic, the trend of using bimetallic nano structures has become popular these days. Mainly, there are two metallic elements present in bimetallic nano structures and generally, their characteristics tend to variate which depends on their metallic composition and structure. Due to a number of applications of the structures of bimetallic nano particles, they can be used in fuel cells in the form of electro-catalysts and can also be used for the purpose of breast and bone surgery. Here, the best example which can be used in these kinds of applications is Ag-Pt structures. There are many synthetic techniques are used in order to fabricate the bimetallic nanostructures which mainly comprises the reduction in alcohol, reduction in citrate and other reduction such as photolytic reduction, and radiolytic reduction.

Also, the synthesis of mono-metallic nano particles can be done with the help of extracts of tea leaves. It was reported that the iron can be reduced by using the tea polyphenols. On the other hand, nano structures can be forms from Palladium and silver ions by using the tea polyphenols. Similarly, Nano scale materials are used in order to address the various problems in the field of nano medicine where a lot of problems arise during the diagnosis and the treatment of the diseases. Also, in recent years, there has been the evolution of certain nano scale materials which can be further used for cancer therapy. The usage of nano structures in cancer therapy is 
increasing year by year due to their property of size control and proper functionality. In cervical cancer, the malignant tumour is found and it is mostly found in females. There is great demand of agents which can be used as cancer therapy assistant tools. On comparing the monometallic nano structures with the bimetallic nano structures, it was found that the physical and chemical properties of the latter are excellent. Whereas, the biological properties of bimetallic nano structures were not found to extraordinary on comparing these with that of monometallic nano structures and compositions.

Here, we use the tea polyphenol as the reducing agent which can be used to prepare the nano structures of Ag-Pt and this process seems to be less costly and also environment friendly as compared to the other processes and methods. Here, MTT assay was used to examine the cells of cervical cancer with the help of the synthesized nano structures of Ag-Pt where its cytotoxic effects were considered. In the field of nano science of nanotechnology, the preparation of nano structured materials is found to be very interesting with respect to its various applications in sensors, solar cells, drug carriers, scaffolds and LED. The usage of green methods while the fabrication of nano particles has motivated the other methods which tend to be eco-friendly and least costly; thus; making these procedures more feasible in nature.

Year by year, the cases of cancer are increasing with a rapid growth and the amazing thing is that there are many limitations in the current method of diagnosis and treatment of cancer. There are many traditional methods which can be used for the treatment of cancer. Some of these methods are radiotherapy, surgery and chemotherapy. There are many drawbacks in the conventional way of therapy where a lot of pain, fatigue, cell damage and anxiety are observed during the treatment. Hence, the nano technology was introduced in order to overcome these drawbacks of traditional way of cancer therapy. Also, the cancer therapy with single drug and combination therapy were found with more efficiency and lesser side-effects. The efficiency of cancer therapy mainly depends on the physical, chemical and biological properties of nanostructures. Here, we tried to fabricate the bimetallic nano structures of Ag-Pt for the reason of chemopreventive of cancer.

\section{MATERIALS AND METHODS}

\section{Materials}

Silver chloride $(\mathrm{AgCl} 2)$ and chloroplatinic acid $(\mathrm{H} 2 \mathrm{PtCl} 6.6 \mathrm{H} 2 \mathrm{O})$ were of analytical grade and used as received. In the experiment, tea polyphenol was also used.

\section{BIMETALLIC Ag-Pt NANOSTRUCTURESSYNTHESIS}

In the preparation of the bimetallic nano structures of Ag-Pt, the quantity of silver chloride was $25 \mathrm{~mL}$ of $1 \times 10^{-3}$ $\mathrm{M}$ and the quantity of $\mathrm{H}_{2} \mathrm{PtCl}_{6}$ solution was $25 \mathrm{~mL}$ of a $1 \times 10^{-3} \mathrm{M}$. Finally, these were mixed with the aqueous solution of tea polyphenol of $5 \mathrm{~mL}$ at some moderate temperature. After half an hour, it was observed that the colour of the solution started changing which indicated that the bimetallic nano structures were formed. Then, these products were allowed to purify at the frequency of $12000 \mathrm{rpm}$ for about 5 minutes. Then, the sample was washed two times with water.

\section{CHARACTERIZATION}

\subsection{Physicochemical Characterization}

Ultraviolet spectroscopy was used in order to analyze the optical properties of the synthesized nano structures of Ag-Pt. Here X-ray diffraction methods were used to examine the crystalline behaviour of nano structures. Also, the transmission electron microscope was used to examine the morphology of nano structures which was used in association 
with the x-ray analysis of energy dispersed. Before analysis, the synthesized bimetallic nanostructure of Ag-Pt was kept on the copper grid coated with carbon. This nano structure was then dried fully in a vacuum desiccator.

\subsection{Cell Culture}

The $\mathrm{SiHa}$ cells were allowed to culture as a mono-layer at $70-80 \%$ confluence comprising $1 \%$ penicillin and $10 \%$ bovine serum. These cells were then used in the experiment.

\subsection{Cell Viability Assay}

The examination of viability of cells of the nano structures of Ag-Pt was performed with the help of MTT assay. These SiHa cells were then allowed to seed in 96 well plates having a density of approximate 1 x 104 cells per well. Then, these cells were exposed to several concentrations $(0,12.5,25,50,100$ and $200 \mu \mathrm{g} / \mathrm{mL})$ of Ag-Pt nanostructures for 24 and $48 \mathrm{~h}$. Then, $20 \mu \mathrm{L}$ of an MTT dye was mixed in each well. Then, these plates were kept at dark place for about 4 hours at the temperature of 37 degree. The absorbance was monitored at $570 \mathrm{~nm}$ (measurement) and $630 \mathrm{~nm}$ (reference) using a 96-well plate reader.

\subsection{RESULTS AND DISCUSSIONS}

\subsubsection{Synthesis of Ag-Pt Nanostructures}

It illustrates that the fabrication of Ag-Pt nano structures with the help of reduction of the ions of platinum and silver by using tea polyphenol. The mixing of the ions of silver and platinum was done and tea poly phenol was mixed into this solution. Generally, there are two phases of the growth process of nano particles of silver and platinum. First is the growth of primary nano particles and the second is the preparation of the secondary nano particles. Similarly, the reduction of metal ions of silver and platinum was carried out to form the nano particles of these metals. As a result, the nano particles were mixed together and the deposition of the nano particles of silver was carried out on the nano structures of platinum. The entrapping of the nano structures of platinum was done with the help of silver nano particles through the synthesis of the secondary nano particles.

\subsubsection{Physicochemical Characterization}

In Figure 4.2, it is shown that the bimetallic nanostructures of silver and platinum showed a wide absorption peak at approximate $400 \mathrm{~nm}$ with respect to the resonance of the surface plasma of the nano particles of silver. Also, the peak intensity tended to decrease as the reaction time increased which indicated that the number of nano particles of platinum slowly increased. In figure 4.3, the FT-IR spectra of tea poly phenol is shown which was observed before and after the reduction of metal ions of silver and platinum. The tea polyphenol shows the absorption peaks at 1694, 1607, 1517, 1450, $1309,1227,1184,1140,1087,1011,866,819,762,730$ and $703 \mathrm{~cm}^{-1}$. 


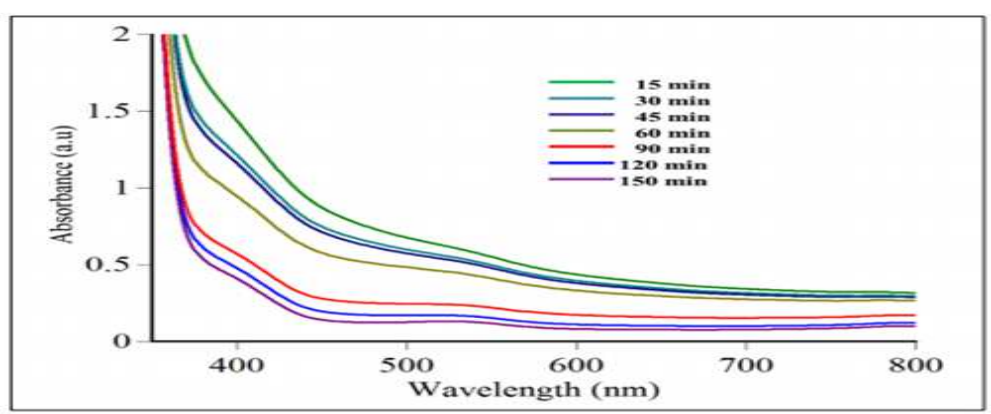

Figure 4.2: UV-V is Spectra of the Prepared Ag-Pt Nanostructure at Different Time intervals

Among these data, the peak of aromatic ring $-\mathrm{C}=\mathrm{C}-\mathrm{C}=\mathrm{O}, \mathrm{C}=\mathrm{C}$ was observed to be $1694 \mathrm{~cm}^{-1}$, the peak of aromatic ring -C-C- was 1517 and that of esters was 1309 and polyols was $1227 \mathrm{~cm}^{-1}$.

Here, the peak of catechins polylols was observed to be $1227 \mathrm{~cm}^{-1}$. C-H alkanes had the peak of 1450 and the same was observed to be 1140 and 1087 in case of $-\mathrm{OH}$ aromatic and $\mathrm{C}-\mathrm{OH}$ alcohols.

Some kind of similarity can be seen in the spectra of nano structures of silver and platinum and tea polyphenol.

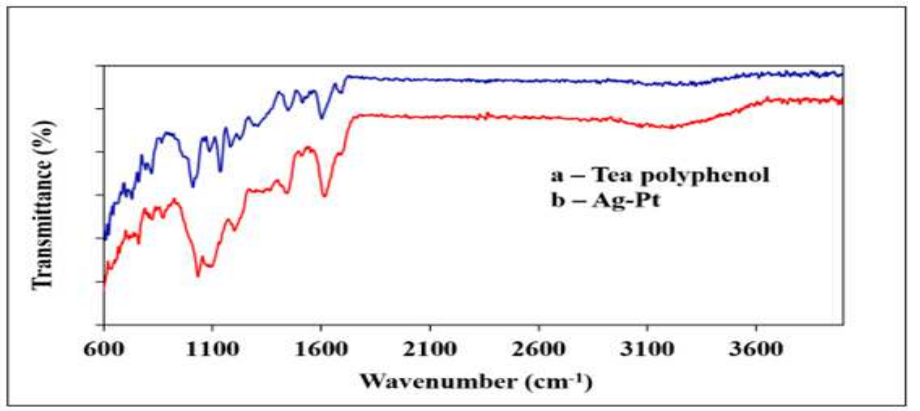

Figure 4.3: FT-IR Spectra of (a) Tea Polyphenol and (b) Ag-Pt Nanostructure

XRD was used to characterize the crystalline structure of synthesized bimetallic nano structures of Ag-Pt which can be shown in figure 4.4. The diffraction peaks were observed in XRD pattern of the nano structures of Ag-Pt ranging from the values of $38.5^{\circ}, 46.1^{\circ}$ and $67.4^{\circ}$. The platinum nano particles were observed to be cubic and face-centred having a peak at $2 \theta=46.1^{\circ}(200)$ and $67.4^{\circ}(220)$. The formation of the nano structures of Ag-Pt was indicated with the help of these peaks as the results evaluated. Here, the microscopy of electron transmission was used to measure the dimensions of the synthesized nano structures.

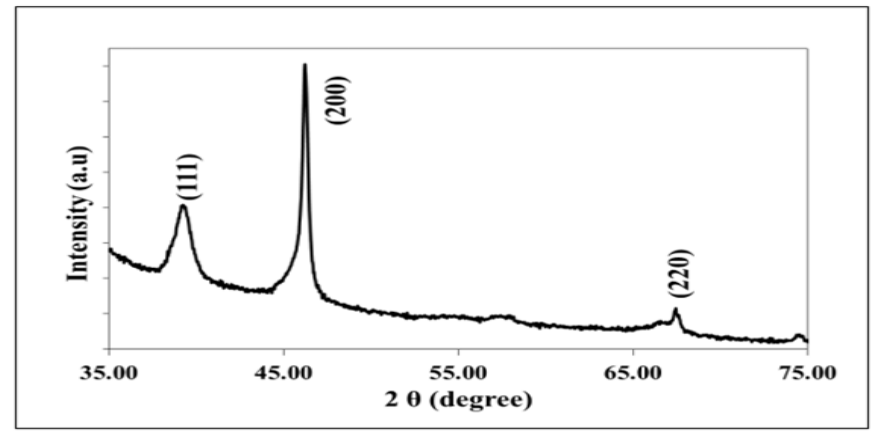

Figure 4.4: XRD Pattern of the Ag-Pt Nanostructure Prepared Using Tea Polyphenol at Ambient Temperature 
In figure 4.5, different scales are used in order to represent the TEM images of the synthesized nano structures. The size of the TEM images of the nano structures of Ag-Pt was observed to be 50-75 nm and the entrapping of silver nano particles and platinum nano structures was also observed.

The figure $4.5 \mathrm{c}$ presents the crystalline image of HRTEM of nano structures of silver and platinum. Here, the assistant of the lattice fringes is done in order to demonstrate the full image.

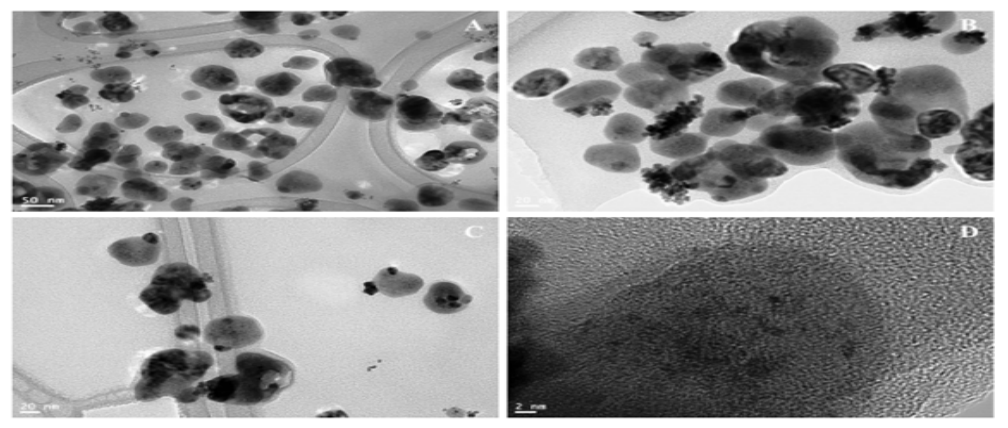

Figure 4.5: TEM Images at (a) Low and (b) High Magnifications and (c) HRTEM Pattern of the Ag-Pt Nanostructure Prepared Using Tea Polyphenol

In the study of synthesized nano structures of Ag-Pt and its composition with the help of TEM with the association of the setup of EDX and the results indicated that $\mathrm{Ag}, \mathrm{Pt}, \mathrm{Cu}$ and $\mathrm{C}$ are present here. There were elements like copper and carbon with respect to the copper grids which was coated with carbon. It also concluded that the synthesized nano structures were in purified form. As a result of XRD and TEM, it was concluded that tea polyphenol has the tendency to help in the reduction of silver and platinum ions to form the nano structures of Ag-Pt. Here, the extracts of green tea were used in order to derive the tea polyphenol having catechins like (-)-epigallocatechin and (-)-epigallocatechingallate. Metals are obtained by reducing the corresponding metal ions by using polyphenols. It is also observed that the ions of silver and platinum has the tendency to form the nano structures of these ions through the usage of tea polyphenol. Also, the mono-metallic nano structures like $\mathrm{Ag}, \mathrm{Pt}$, Au etc. can be formed by reducing the metal ions by using the tea extracts and agents. MTT assay was used to measure the cyto-toxic properties of the nano structures of Ag-Pt over the cells of $\mathrm{SiHa}$. It is shown that the SiHa cells were allowed to expose to the nano structures of the Ag-Pt at various concentrations $\mathrm{i}$. e. $(12.5,25,50,100$ or $200 \mu \mathrm{g} / \mathrm{mL})$ for 24 and $48 \mathrm{~h}$. On increasing the concentration level for Ag-Pt nanostructures, some changes were observed in the viability of cells after an exposure of about 24 hours which certainly indicated the dependency of these SiHa cells on the doses.

It was also observed that on enhancing the exposure time from 24 to 48 hours, the cells started showing a significant decrease in the level of viability which was from $18 \%$ to $16 \%$ i. e. a fall of about $2 \%$ was noticed on increasing the exposure time. These results showed that time and doses play an important role in the induction of SiHa cell death. Other studies suggested that the death of cells is induced by nano particles which are dependent on their characteristics like surface area, dose and exposure time. Apoptosis and Necrosis are two significant factors for cell death. Necrosis is responsible for the cell death through outer cracks done by the damage of the membrane of plasma which favours its level of integrity and characteristics. On the other hand, Apoptosis is responsible for the programmed cell death, which is initiated by shrinking of cells, condensation and decomposition of DNA. The results of the study indicate that apoptosis play a role in the death of the cells by inducing the nano structures of Ag-Pt. 
More and more research works need to be done and investigated, in order to explore the properties of the nano structures of Silver and Platinum. These properties may include the molecular, functional, optical, physical and chemical properties. Further research work is needed and suggested in this work so that the proper information can be obtained regarding these nano structures. Here, flow cytometry was used in order to analyze themolecular mechanisms which was further based on the particular cycle of the natural effects of the nano structures of silver and platinum. This mechanism depends on the quantitative measurements of the cells and their nuclear content of DNA. Flow cytometry was also used to analyze the cells which were allowed to react with the nano structures of silver and platinum $(25 \mu \mathrm{g} / \mathrm{mL})$ for $24 \mathrm{~h}$ after PI staining.

Here, Apoptosis was used in order to trigger the Ag-Pt nano structures within the cells of cervical cancer. These results will help in the further development of therapeutic techniques for the preservation and cervical cancer treatment and also Ag-Pt nano structures can be used in the traditional anti-cancer therapies. It is also observed that the non-specificity of the drugs can be reduced by siting the targeted tumor. Sometimes, the cell membranes and their surface receive the several varieties of protein. Hypdrophilic characteristics play a significant role with respect to the type of agent and nanoconjugate size which is essential for the better therapeutic efficacy. These nano-particles impose higher potential which can be used for medical applications. However, the interaction knowledge between the cells and its mechanism can be tracked in the human body but still, there are many issues which make it difficult to happen. Hence, many researches are going on to make this tracking easier, so that the scope of the nano particles can be enhanced. Now, it is possible to examine the interactions of the nano-particles at the molecular scale through cells. This is also usable for the structure of nanoconjugates which can be used to struggle several vital diseases which were supposed to be uncured and hence, the conditions in the health sector have begun to improvise with the introduction of nano-science. Cisplatin which is known as a derivative of Platinum is also used for cancer therapy. The platinum complexes are used for the death of tumor cells where the drugs are used as a spectrum. Platinum nano particles are also used for breast cancer, neck cancer and stomach cancer etc. The anti-cancer properties of Platinum form a base to use it in the form of anti-cancer so that the utility of metallic nano particles can be enhanced.

These nano-particles of Platinum show their resistance towards the cancer cells and myelogenous leukemia can be used in order to treat the cancer cells which are supposed to be sensitive in nature. In a study, the dose of lobaplatin was usedfor the treatment of tumor where docetaxel was mixed with lobaplatin and this mixture was then used for tumor treatment. This mixture was further used for chemo-therapy. In another case, Picoplatin was used to facilitate the platinum to prevent the attacks from nucleophiles which resulted in the hindrance of the steric. This also can be used as protective shield which can be used to repair the DNA. Preliminary observations suggested that cisplatin can be used for anti-cancer activities. Also, picoplatin works as main cell line in spite of a fall in neuro toxicities. Picoplatin can also be used with the mixture of fluorouracil and leucovorin which can be served as the anti-cancer agent in the human body. The drugs like Satraplatin and dichloro-(cyclohexylamine) platinum can be used orally. It shows several characteristics regarding other compounds of platinum and therefore, it may show a several vision of resisting cancer. Satraplatin can also be used for the treatment of several cancer like cervical, lung and ovarian cancer etc. In preliminary observations, the usage of satraplatin was abandoned in non-linear pharmaco-kinetics. Many clinical trials were performed in order to undergo Satraplatin in human body against refractory cancer. The cancer cells were also targeted with the help of Lipoplatin where safety was maintained by cisplatin which s supposed to be a form of Platinum. These liposomes are comprised of cholesterol and soyphosphatidyl choline. The toxicity was made to fall down through the inhibitory effect in case of normal cells which is 
essential for the stability of the concentration of plasma. Some Platinum nano particles are used for the execution of anticancer activities so that their cyto-toxic features can be used to prepare the platinum compounds for the better management of the platinum drugs and doses.

However, the scientific results are yet to be observed to know the consistency of these nano particles for the treatment of cancer from medical applications providing the information regarding the safety profiles of the used drugs and some socio-economic factors are needed to be considered. In some cases, the lack of activities of resisting the cancer and toxicity proved to be vital as some pattern of discontinuation in the platinum nanoparticles was observed. An alternative to cisplatin was attempted by the screening process of drugs primarily based on platinum where the resistance for cancer was observed because of adverse effects which arose due to dose. A number of Platinum drugs were thus eliminated from the trials and these drugs included miboplatin, zeniplatin and ormaplatin JM 11 and NSC 17089812. Also, conjugated platinum(II) was used for the drug delivery of the platinum drugs into the cells so that a proper transportation for the drug transmission can be designed.

The reason behind it was that it was essential to facilitate the maintenance of cellular homeostasis for the growth of healthy cells which can show an upper rate of glycolysis in case of healthy cells otherwise it seems difficult to maintain the proper growth. The transportation of nanoparticles of Platinum was made easier through the docking analysis of related molecules so that the structure of the particles can be maintained and it can effectively be used as an anti-cancer agent. It is also observed that the Platinum nanoparticles can be used to conjugate the complexes and substrates which may be helpful for the evaluation of cyto-toxicity. Also, the efficiency and stability of conjugates of platinum nanoparticles was studied in order to analyze the stability of these nano particles which can be used for the development of drugs and other research work related to pharma industry. This concluded that A2780 cells were sensitive in nature and these required some kind of inhibitory concentration within the range of $0.15-0.22 \mu \mathrm{M}$. These Platinum nanoparticles also have the tendency to bind to DNA through only one coordination end. These nanoparticles can also be synthesized by just showing the cyto-toxic effects which resisted the cancer cells. On the comparison of Phenanthriplatin with cisplatin, it was observed that the former showed improved cyto-toxic effect. The mono-functional adducts are responsible for the biological activities of these particles.

\section{CONCLUSIONS}

We developed a synthetic method to fabricate the nano structures of Ag Pt. This synthetic method is the best option which can be used in place of other physical and chemical methods. The role of reduction agent was done by tea poly phenol. Here, tea poly phenol was used to perform the reduction of ions of metal to the nano structures of Ag-Pt. The results of powder XRD indicated the structure of crystalline stage of $\mathrm{Ag} \mathrm{Pt}$ to be the cubic facing towards center. The size of nano structures of Ag-Pt was observed to be approximate 50-75 nm and transmission electron microscopy was used to get the measurements. Thus, the size of synthesized spherical and dispersed nano structures of Ag-Pt was evaluated. The EDX results also indicated that the nano structures of Ag-Pt were pure in nature. It was also observed that the death of cells in Siha cells were induced by the nano structures of Ag-Pt. The cytotoxic effects were measured with the help of colometric assay of MTT. The death of cells in SiHa cells were caused by the nano structures of Ag-Pt. Also, the decomposition of DNA was observed by the Ag-Pt nano structures. Also, an increase in the number of cells in SiHa cells was observed with the introduction of nanostructures of Ag-Pt in the cycle of cells. Hence, it was concluded that Ag-Pt nanostructures with the collaboration of tea polyphenol can be used for the purpose of cancer treatment. 


\section{REFERENCES}

1. Min Y, Li J, Liu F, Yeow EK, Xing B. NIR light mediated photoactivation pt based antitumor prodrug and simultanous cellular apoptosis imaging via upconversion nanoparticles. Angew Chem Int Ed Engl. 2014;53:1012-6.

2. Rout SR, Behera B, Maiti TK, Mohapatra S. Multifunctional magnetic calcium phosphate nanoparticles for targeted platin delivery. Dalton Trans. 2012;41:10777-83.

3. Wang J, Wang X, Song Y, Zhu C, Wang K, Guo Z. Detecting and delivering platinum anticancer drugs using fluorescent maghemite nanoparticles. Chem Commun (Camb). 2013;49:2786-8.

4. Peddi, S. P., \& Sadeh, B. A. (2015). Biosynthesis of silver nanoparticles using Achyranthes aspera L. stem extract. Int. J. Phys. Res, 5(1), 41-54.

5. Yoshihisa Y, Zhao QL, Hassan MA, Wei ZL, Furuichi M, Miyamoto Y, Kondo T, Shimizu T. SOD/catalase mimetic platinum nanoparticles inhibit heat-induced apoptosis in human lymphoma U937 and HH cells. Free Radic Res. 2011,45:326-35.

6. Kajita M, Hikosaka K, Iitsuka M, Kanayama A, Toshima N, Miyamoto Y. Platinum nanoparticle is a useful scavenger of superoxide anion and hydrogen peroxide. Free Radic Res. 2007;41:615-26.

7. Porcel E, Liehn S, Remita H, Usami N, Koayashi K, Furusawa Y, Lesech C, Lacombe S. Platinum nanoparticles: a promising material for future cancer therapy? Nanotechnology. 2010;21:085103-10.

8. Habeeb, M. A. (2013). Effect of Nanosilver Particles on Thermal and Dielectric Properties of (PVA-PVP) Films. International Journal of Applied and Natural Sciences, 2(4), 103-108.

9. Wang S, Meckling KA, Marcone MF, Kakuda Y, Tsao R. Can phytochemical antioxidant rich foods act as anti-cancer agents? Food Res Int. 2011;44:2545-54.

10. Marchand L, Murphy S, Hankin JH, Wikens LR, Kolonel LN. Intake of flavonoids and lung cancer. J Natl Cancer Inst. 2000;92:154-60.

11. Cherit-Hacid, F., Derridij, A., Moulti-Mati, F. A. R. I. D. A., \& Mati, A. B. D. E. R. R. A. H. M. A. N. E. (2015). Drought stress effect on some biochemical and physiological parameters; accumulation on total polyphenols and flavonoids in leaves of two provenance seedling Pistacia lentiscus. Int. J. Res. Appl. Nat. Soc. Sci, 3(9).

12. Sun J, Liu RH. Cranberry phytochemical extracts induce cell cycle arrest and apoptosis in human MCF-7 breast cancer cells. Cancer Lett. 2006;241:124-34.

13. Higdon JV, Frei B. Tea catechins and polyphenols: health effects, metabolism, and antioxidant functions. Crit Rev Food Sci Nutr. 2003;43:89. 